\title{
Infection with intracellular parasite Amoeboaphelidium protococcarum induces shifts in associated bacterial communities in microalgae cultures
}

\author{
Anna-Lena Höger ${ }^{1}$ (D . Carola Griehl ${ }^{1}$ (D) Matthias NolI $^{2}$ (D)
}

Received: 21 January 2021 / Revised and accepted 14 June 2021 / Published online: 6 September 2021

(c) The Author(s) 2021

\begin{abstract}
In recent years microalgae products have developed increasing market demand, but sustainable industrial production is still challenged by biological stability of large-scale production plants. Yet the relationships between algal hosts, associated microbiomes, and contaminants in photobioreactors remains widely understudied. The aim of this study was to investigate the temporal development of microbiomes of four freshwater microalgae species Scenedesmus vacuolatus, Desmodesmus quadricauda, Chlorella sorokiniana, and Botryococcus braunii, in presence and absence of the zoosporic parasite Amoeboaphelidium protococcarum. To compare the effects of sterile and nonsterile culture conditions, infection experiments were performed in sterile laboratory (sterile) and simulated industrial conditions (open). Algal growth (dry weight, optical density, and nutrient consumption) was observed for 21 days, and samples of the associated microbiome were collected for bacterial 16S rRNA gene Illumina MiSeq sequencing. Infection patterns of A. protococcarum were algae species-specific, irrespectively of culture conditions. Bacterial community analysis demonstrated distinct and stable bacterial communities for each algae species, which were mostly dominated by $\alpha$ - and $\gamma$-Proteobacteria. Upon aphelid parasitosis, bacterial diversity increased, and community compositions diverged algae-specific over time. Moreover, bacterial functional traits shifted to detoxification, degradation, and cellulolysis once algae were infected. This study provides a first insight into the close connection between algae, associated bacterial microbiomes and appearing contaminants in photobioreactor systems.
\end{abstract}

Keywords Algae-associated microbiome $\cdot$ Algae-bacteria interactions $\cdot$ Algal parasites $\cdot$ Aphelids $\cdot$ Functional diversity $\cdot$ Microalgae

\section{Introduction}

Cost-effective ways to produce large quantities of algal biomass are crucial to exploit the full potential of microalgae plants. Besides high energetic and financial expenses for optimal cultivation conditions, harvesting, and downstream processes, one major challenge to the sustainable production of algae biomass is maintaining biological stability in large-scale production plants (Scott et al. 2010; Kazamia et al. 2012; Wang et al. 2013; Carney and Lane 2014).

Matthias Noll

matthias.noll@hs-coburg.de

1 Competence Center Algal Biotechnology, Anhalt University of Applied Sciences, Koethen, Germany

2 Institute for Bioanalysis, Coburg University of Applied Sciences and Arts, Coburg, Germany
Since sterile operation of axenic cultures at large scale is economically and practically not feasible, the formation of dynamic ecosystems in open ponds and closed photobioreactors (PBRs) is almost inevitable (Schenk et al. 2008; Cooper and Smith 2015). Recent studies have revealed the diversity and dynamics of associated microbiomes and complexity of interactions in artificial microalgae cultivation systems that seemed to be similar to the algae holobiont in natural systems (Lian et al. 2018; Ferro et al. 2020).

Algae are strongly associated with complex microbial communities in natural environments, and their interaction patterns have formed in a long coexisting evolutionary history. The phytoplankton-bacteria relationship represents a fundamental role in ecosystem functioning and has an immense impact on biogeochemical cycling in aquatic environments at a global scale (Azam 1998; Buchan et al. 2014). The microenvironment of the phycosphere allows the exchange of manifold metabolites and chemical compounds 
that can result in a wide spectrum of mutualistic, commensalistic, antagonistic, competitive, or parasitic relationships (Kazamia et al. 2016; Ramanan et al. 2016; Seymour et al. 2017; Cirri and Pohnert 2019). Such algae-bacterial relationships can be altered by abiotic factors like temperature and/or light availability (Mayers et al. 2016; Piwosz et al. 2020). The relationship of algae and associated bacterial communities has to be therefore considered as a dynamic range of subsequent states of highly complex networks influenced by changing environmental conditions (Gurung et al. 1999; Seyedsayamdost et al. 2014; Fuentes et al. 2016; Mayers et al. 2016; Cirri and Pohnert 2019).

Some studies conclude that algae-bacterial interactions are algae species-specific (Krohn-Molt et al. 2017; Behringer et al. 2018; Crenn et al. 2018; Koedooder et al. 2019; Mönnich et al. 2020). However, other studies suggest that these interactions were mainly based on bacterial functional traits (Ramanan et al. 2015; Geng et al. 2016a). Bacterial functional traits can be gained from 16S rRNA gene-based approaches by FAPROTAX, which was introduced for ocean environments and was recently improved and extended also for other environments (Sansupa et al. 2021).

In the past, bacterial communities were often considered as mere contaminants that can inhibit algae productivity or terminate algae populations. However, recent findings have shown positive effects of algae-bacterial interactions considered promising for biotechnological applications. The potential benefit can be found in enhanced biomass production, simplified harvesting, wastewater treatment, and bioremediation for sustainable aquaculture (Wang et al. 2014; Fuentes et al. 2016).

Apart from bacterial communities, algae also have to face fungus-like parasites, which pose a high threat for plant managers as they cause a sudden and massive death of microalgal cells in natural environments as well as in industrial systems (Gachon et al. 2010; Carney and Lane 2014). The mycoloop, driven by such zoosporic parasites, has a strong ecological impact on aquatic food web dynamics by regulating phytoplankton population size and providing access of nutrients to higher trophic levels (van Donk 1989; Sime-Ngando 2012; Kagami et al. 2014; Rasconi et al. 2014; Frenken et al. 2017; Jephcott et al. 2017). The family Aphelidiaceae represents a poorly known group of obligate endoparasitoids of various algae. The group was recently affiliated to the superphylum Opisthosporidia, but the phylogenetic relation to fungi is still under active discussion (Karpov et al. 2014; Tedersoo et al. 2018; Torruella et al. 2018). Hitherto, four genera (Aphelidium, Amoeboaphelidium, Pseudaphelidium, Paraphelidium) with 17 species have been described (Letcher and Powell 2019; Karpov et al. 2020; Seto et al. 2020). The distinct morphological feature in the complex lifecycle of aphelids has been determined as an intercellular amoeboid state. The detailed life cycle of Amoeboaphelidium protococcarum has been characterized by Letcher and colleagues (2013). Briefly, pseudopodiate aplanospores of $A$. protococcarum attach to intact host algal cell walls and form encapsulated cysts. Thereafter, penetration tubes are formed by the cysts to enter as ameboid trophont into the algal cytoplasm. The trophont engulfs the algal cytoplasm by phagocytosis, leaving a residual body. After division, a mature sporangium releases new flagellated zoospores of A. protococcarum.

The host specificity of $A$. protococcarum comprises protococcous algae, including various Scenedesmus species (Gromov and Mamkaeva 1968, 1969; Schnepf et al. 1971). Furthermore, different algae species or even strains exhibited varying sensitivity to different $A$. protococcarum strains (Gromov and Mamkaeva 1969). In 2017, a new isolate of A. protococcarum (WZ01) was identified causing an algal population collapse in an open raceway pond (Ding et al. 2017). Additionally to some unusual morphological characteristics, shifted pathogenicity patterns towards coccoid green algae were observed in the WZ01 isolate. These results underline that a comprehensive understanding of host specificity and the underlying mechanism of how aphelids detect and differentiate host cells is still not reported.

Infections with zoosporic parasites can lead to rapid and complete biomass loss in large-scale algal plants, which has been reported repeatedly in literature in recent years (Hoffman et al. 2008; Letcher et al. 2013; Carney et al. 2014; Ding et al. 2017). As commercial scale culture systems tend to be unialgal, specific parasites have a devastating effect on mass monocultures. Contamination management is still at an early stage, but promising methods of synthetic ecology and community engineering that manage microbial diversity and species composition leading to increased resilience and productivity simultaneously come to the fore (Shurin et al. 2013; Smith and Crews 2014; McBride et al. 2016). Hence, it is crucial to further investigate and understand the co-occurrence and microbial associations in artificial microalgae systems to accelerate such approaches (Mendes and Vermelho 2013; McBride et al. 2014; Carney et al. 2016).

Objectives of this study were (i) to investigate the temporal development of bacterial microbiomes within cultures of different algae species; (ii) to compare the effects of sterile laboratory conditions (sterile) and nonsterile culture conditions usually applied in industrial production processes (open) on the bacterial community composition; and (iii) to assess whether, or to what extent, associated bacterial communities are affected if the respective algae has been infected by aphelids $(+\mathrm{AI})$. 


\section{Material and methods}

\section{Algae/parasite culturing and experimental design}

Algae cultivation was conducted in closed photobioreactors at the Competence Center Algal Biotechnology in Koethen, Germany, in 2017/2018. All experiments were performed in bubble column reactors (BCR) with $1.5 \mathrm{~L}$ capacity and autoclaved modified Bolds basal medium (BBM) (Bischoff and Bold 1963) at $23.5^{\circ} \mathrm{C}$, a gas flow of 1.0 volume of air per culture medium $\left(1 \% \mathrm{CO}_{2}\right)$ and permanent illumination at $100 \mu \mathrm{mol}$ photons $\mathrm{m}^{-2} \mathrm{~s}^{-1}$ with a daylight white LED light (380-750 $\mathrm{nm})$.

Experiments were conducted with the algae strains Scenedesmus vacuolatus SAG 211-8b, Desmodesmus quadricauda CCALA 464, Chlorella sorokiniana SAG 211-8 k, and Botryococcus braunii var. Showa. In each case, BCRs were inoculated to an optical density (OD) of 0.2 at a wavelength of $750 \mathrm{~nm}$ under sterile laboratory conditions (sterile; handling in a laminar flow box) and open "industrial" conditions (open; handling on a workbench), each with 6 replicates. Three independent replicates of each algae culture were thereafter infected with $100-\mathrm{mL}(6.6 \% \mathrm{v} / \mathrm{v})$ aphelid inoculum $(+\mathrm{AI})$, while $100-\mathrm{mL} \mathrm{ddH}_{2} \mathrm{O}$ was added to additional three independent replicates as negative control cultures (-AI). Each algae culture of the four resulting culture treatments (sterile + AI, open + AI, sterile -AI, open -AI) was sampled every $48 \mathrm{~h}$ for a period of 21 days and immediately checked for growth parameters. Dry weight content was determined by gravimetric measurement of dried biomass (Moheimani et al. 2013), and $\mathrm{OD}_{750 \mathrm{~nm}}$ was detected on a Tecan microplate Reader (Tecan; Switzerland). Nutrient consumption was monitored by ion chromatography using a Dionex ICS 1100 system (Thermo Scientific; USA) according to manufacturers' instructions. The morphological status of algal cells was evaluated and documented by using bright-field microscopy with an Olympus BX41 microscope and Olympus XC50 camera. Retained samples for nucleic acid-based analysis of the bacterial community structure were stored at $-80^{\circ} \mathrm{C}$ until further processing.

An enrichment culture of $A$. protococcarum was obtained from a contaminated Christmas tree bioreactor at the Biosolar Center in Koethen, which was described previously (Matthes et al. 2015). Strain A. protococcarum AI15TR was isolated through repeated serial dilution and plaque plating on BBM agar plates as explained earlier (Letcher et al. 2013), and the elimination of other microorganisms was assessed by microscopic observations and cultivation with R2A selective agar as described earlier (Gibbs and Hayes 1988; Andersen 2015). The resulting aphelid stock cultures were stored at $-20{ }^{\circ} \mathrm{C}$ for longer periods without losing the infectivity to $S$. vacuolatus. To prepare aphelid inoculum, axenic cultures of $S$. vacuolatus were grown to mid-log phase in BBM for 5 days, diluted to a final $\mathrm{OD}_{750}$ of 0.2 in $1.4 \mathrm{~L}$, infected with $100 \mathrm{~mL}$ purified A. protococcarum stock and cultivated in BCRs using the same culture parameters applied in the following experiments. Seven days after infection, cultures were harvested, microscopically checked for infection status and thereafter frozen for later use. For all contamination experiments described in this publication, the same bulk volume of aphelid inoculum was used to secure consistent composition, cell density, and pathogenicity of $A$. protococcarum AI15TR.

\section{DNA extraction and amplicon sequencing}

After 0 days, 8 days, and 16 days of incubation of each treatment, samples were collected, and independent replicates of the samples were pooled in equimolar amounts. One sample of the aphelid inoculum was analyzed alongside the algae samples. Genomic DNA was extracted according to Letcher et al. (2017) using the DNeasy Powersoil Kit (Qiagen; Netherlands) following the manufacturer's protocols. Quantity and quality of nucleic acid extracts were checked and sent to Microsynth AG (Balgach, Switzerland) for subsequent amplicon sequencing. Two-step Nextera PCR libraries of the V5 to V7 regions of the bacterial 16S rRNA gene were employed by using the primer pair 799F (5'-AACMGGA TTAGATACCCKG-3') and 1139R (5'-ACGTCATCCCCA CCTTCC-3') (Beckers et al. 2016). Subsequently a v2 500 sequencing run on the Illumina MiSeq platform was performed according to the manufacturer's instructions (Illumina; USA) by Microsynth AG (Balgach, Switzerland).

\section{Sequencing data analysis}

Bacterial 16S rRNA gene sequencing data were processed according to Buettner and Noll (2018) resulting in a final absolute OTU table. OTU sequences were compared against the reference sequences of the SILVA 16S database, and taxonomies were predicted using SINA aligner (v1.2.11), considering a minimum confidence threshold of 0.7 (Pruesse et al. 2012). Bacterial OTUs that could not be affiliated with a higher resolution are hereafter referred to as "unknown bacteria". A MUSCLE alignment (Edgar 2004) was calculated based on the neighbor-joining method (Saitou and Nei 1987) in MEGA X (Kumar et al. 2018). The alignment was used to compute a phylogenetic tree with the maximum likelihood method using the general time-reversible model (Waddell and Steel 1997). Relative abundances were calculated on basis of the absolute OTU table and used for subsequent analyses. 
Fig. 1 Analysis of dry weight biomass content of $S$. vacuolatus, D. quadricauda, C. sorokiniana, and B. braunii cultures over 21 days of incubation. Comparison of infected $(+\mathrm{AI})$ and noninfected cultures (-AI) in open and sterile conditions are shown respectively in the legend (mean of $n=3 \pm \mathrm{SD}$ )
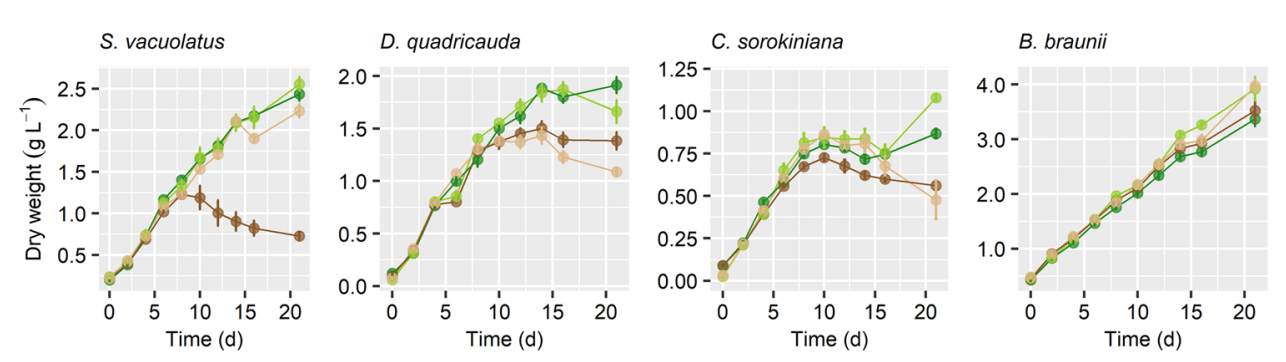

Treatment $\rightarrow$ open-Al $\rightarrow$ open $+\mathrm{Al} \rightarrow$ sterile-Al $\rightarrow$ sterile+Al

$$
\text { . }
$$

\section{Bacterial community composition}

Composition and structure of bacterial communities based on sequencing data were analyzed using the bioconductor package phyloseq (v 1.28.0) and visualized with the implemented package ggplot2 (v3.3.2) on the open-source platform R (v3.6.1). Calculations of alpha diversity measurements were conducted using the Shannon index as an estimator of species richness and evenness, while Chaol was used for estimating the number of species in a community based on the low abundance species (Kim et al. 2017). To determine the structure of the bacterial community composition, Bray-Curtis dissimilarity and weighted UniFrac distance metrics were computed and visualized by nonmetric multidimensional scaling (NMDS). While Bray-Curtis dissimilarity includes only abundance data, the weighted Unifrac metric also includes phylogenetic information to calculate distances (McMurdie and Holmes 2013). In addition, a PERMANOVA test (ADONIS) was conducted in the vegan package (Oksanen et al. 2019) to statistically test the effect of algae species, incubation time and culture treatment on the bacterial community structures. Relative OTU abundances of the bacterial communities on genus level were visualized by displaying the most abundant 20 genera. Rare genera, which accounted for $<1 \%$ each in each algae data set, were merged into the category "Accumulated $<1 \%$ ". Furthermore, numbers of shared and unique OTUs present in the bacterial community composition of different sample types (algae species or culture treatments) were determined by Venn diagrams (Larsson 2020).

\section{Microbial community function analysis}

Metabolic and ecologically relevant functions were retrieved from the FAPROTAX database (Louca et al. 2016) by using the MACADAM explore web interface at genus level (Le Boulch et al. 2019). Subsequently, functions from all bacterial genera were compiled per sample and assigned to different groups (algae species and culture treatments). Finally, the percentage of each function for each group was calculated with Excel (Microsoft, USA) and plotted with the ggplot2 package on $\mathrm{R}$.

\section{Results}

\section{Amoeboaphelidium protococcarum decreased algal growth parameters}

Dry weight, optical density, and nutrient consumption of all noninfected algae cultures (-AI) continuously increased over time irrespectively of culture conditions (open vs. sterile) (Fig. 1, Table S1-3). On the other hand, an algal species-specific decrease in the same parameters was observed after aphelid infection (+ AI) (Fig. 1, Table S1-4). Morphological changes of algal cells like attached cysts, chloroplast reduction, and residual bodies as typically induced by intracellular parasitosis of A. protococcarum were observed in infected S. vacuolatus, D. quadricauda, and C. sorokiniana, but not in B. braunii (Fig. S1).

\section{Bacterial community compositions were shaped by algal host and diverged after aphelid infection}

Bacterial community structures were shaped by the respective algal host as determined by weighted UniFrac (Fig. 2, Table 1) and Bray-Curtis distances (Fig. S2, Table 1). Overall bacterial composition within one algae host was similar to each other at the beginning of the experiment but significantly diverged over time (Fig. 2). Aphelid infection had a greater impact on shifts of the bacterial community composition compared to culture conditions (Fig. 2). Both Shannon and Chaol indices increased over time for all aphelid infected algae cultures $(+\mathrm{Al})$ regardless of culture conditions (open vs. sterile conditions) (Fig. S3). Bacterial diversity was lower in algae cultures without aphelid infection compared to those with aphelid infection (Fig. 3). Culture conditions had only a minor effect on the bacterial diversity associated with $D$. quadricauda and B. braunii, but bacterial diversity was more complex in open culture conditions when associated with S. vacuolatus and C. sorokiniana (Fig. S3), especially in noninfected cultures.

The overall bacterial composition was dominated by members of the phyla $\alpha$-Proteobacteria, $\gamma$-Proteobacteria, and $\delta$-Proteobacteria (for details see Table S5). The 


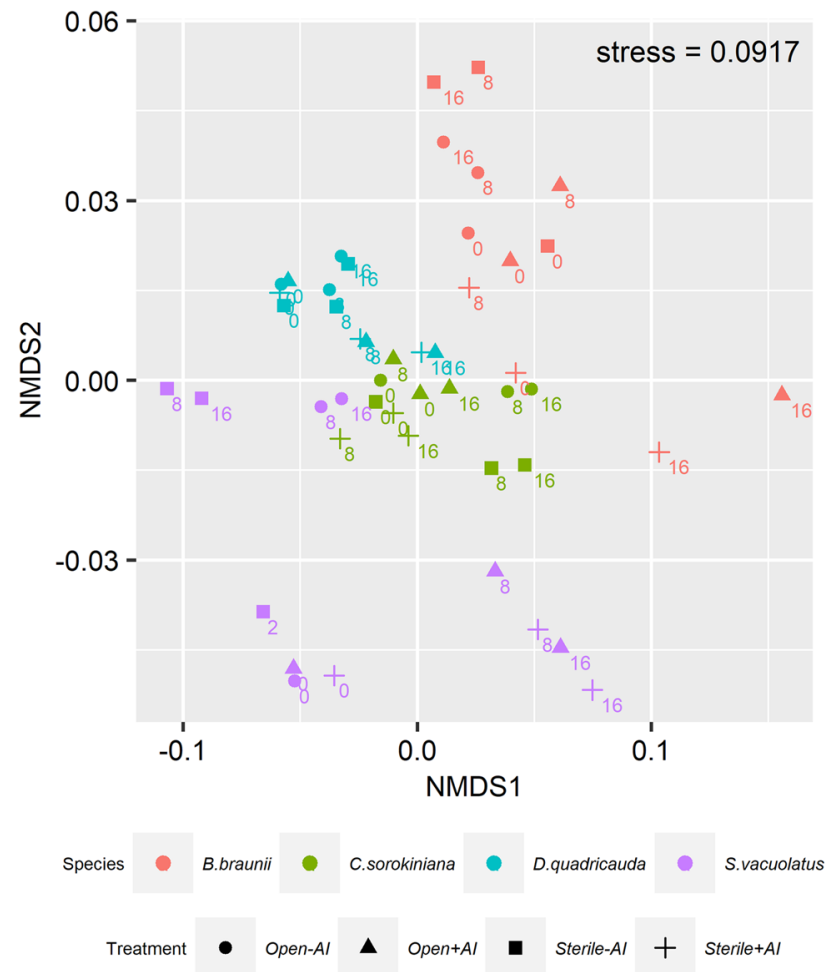

Fig. 2 Nonmetric dimensional scaling (NMDS) based on weighted UniFrac distances of bacterial community composition retrieved from S. vacuolatus (purple), D. quadricauda (cyan), C. sorokiniana (green) or B. braunii (red). Algae cultures are marked according to culture treatments (circle $(\bullet)$ means open-AI; triangle $(\boldsymbol{\Delta})$ means open-AI; square ( $\mathbf{a})$ means sterile-AI, cross $(+)$ means sterile $+\mathrm{AI}$ ) and sampling times (days $0,8,16$ ). Ordination stress indicates the goodness of fit

profound majority of taxa on OTU level only occurred in samples of one algae species (Fig. S4). In contrast, approx. $48.0 \%$ of bacterial OTUs were present in every algae culture regardless of the treatment. Additionally, 26.8\% of the communities were only found in samples treated with aphelid inoculum (+ AI), while OTUs occurring only in samples of noninfected algae cultures (-AI) accounted for $43.5 \%$.
Although shifts in the bacterial community composition were attributed to aphelid infection and incubation time, several dominant taxa remained stable for the respective algal host (Fig. 3). Likewise, bacterial community composition associated with $S$. vacuolatus was mostly composed of members of the genera Hydrogenophaga, Sphingomonas, and Brevundimonas (Table S5). However, the bacterial community composition of $S$. vacuolatus was less diverse in sterile and open culture conditions without inoculum addition (Fig. 3, Fig. S5). Members of the genus Brevundimonas and Devosia dominated the open and sterile conditions after 2 and 4 days, respectively, and thereafter, the community composition pattern was more diverse. Bacterial community composition associated with $C$. sorokiniana was characterized by members of the genera Porphyrobacter, Brevundimonas, and Allorhizobium-Neorhizobium-Pararhizobium-Rhizobium (ANPR) group. In contrast, bacterial community composition retrieved from $D$. quadricauda was characterized by members of the genera Sphingopyxis, Blastomonas, and Brevundimonas, while B. braunii microbiomes were composed of Porphyrobacter, Sphingopyxis, and Hydrogenophaga (Table S5).

Since the growth of $S$. vacuolatus cultures was significantly less affected by the infection with $A$. protococcarum in open culture conditions, the major differences of open and sterile microbiomes were compared (Fig. 4). Especially relative abundance of Hydrogenophaga increased in open culture conditions whereas, e.g., Pseudomonas and Sphingomonas showed higher relative abundances in sterile culture conditions once A. protococcarum infected S. vacuolatus (Fig. 4).

\section{Aphelids caused shifts in bacterial functional traits}

The bacterial functional traits associated with all algal hosts included photoautotrophic and/or photoheterotrophic members (Table S6), which was understandable in a permanently illuminated, but nutrient-poor environment. In addition, all algal cultures were inhabited by chemoheterotrophic bacterial members. Furthermore, functional traits to metabolize

Table 1 Effects of algal host, incubation time, and culture treatments on the bacterial community composition as revealed by PERMANOVA analysis of the Bray-Curtis dissimilarity and Unifrac distance metrics

\begin{tabular}{lllllll}
\hline Parameters & \multicolumn{2}{l}{ Bray-Curtis } & & & wUnifrac \\
\cline { 2 - 6 } & F-model & $R^{2}$ & $p$ value & & F-model & $R^{2}$ \\
\hline Algae species & 26.6230 & 0.58641 & $0.001^{*}$ & 12.4427 & 0.41055 \\
Incubation time & 2.6394 & 0.05811 & $0.002^{*}$ & 2.5677 & 0.08472 \\
Culture treatment & 3.4921 & 0.07688 & $0.001^{*}$ & 2.6301 & $0.021^{*}$ \\
Residuals & & 0.27887 & & & 0.08678 & 0.41794 \\
\hline
\end{tabular}

Significant differences $(p<0.05)$ are indicated by asterisk $(*)$ 

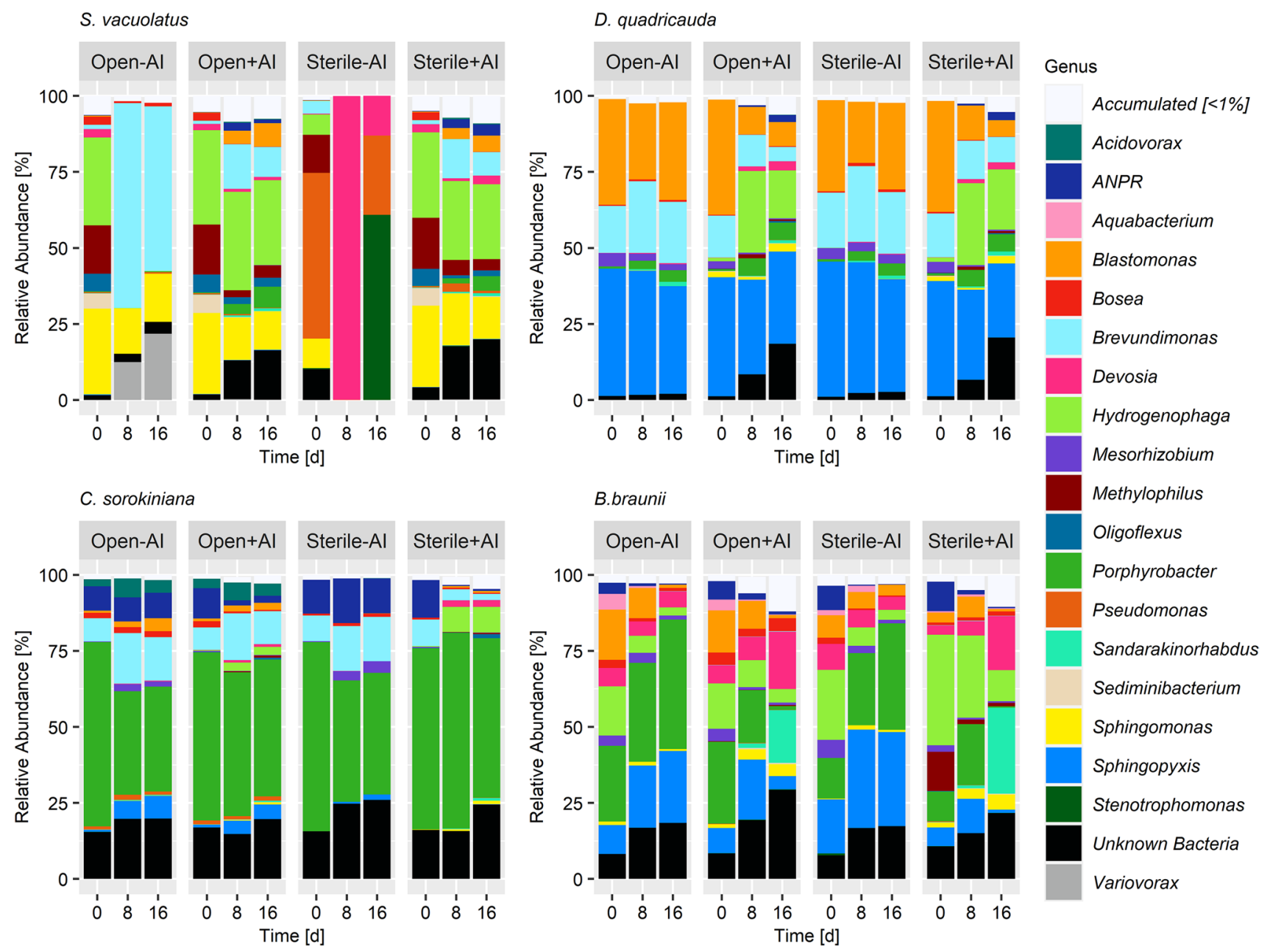

Fig. 3 Succession of bacterial community compositions in four microalgae cultures at genus level. Rare genera, accounting for less than $1 \%$ relative abundance in each algae data set, were summarized in "Accumulated $<1 \%$ ". "Unknown Bacteria" represent OTUs that

mineral nutrients such as manganese, nitrate, sulfur, or iron components, which are key elements in algae culture media, were prominently represented. The overall functional diversity was in great accordance between the algae species (Table S6), despite the compositional differences of the bacterial community. However, if aphelid infection was present, the functional diversity of the bacterial community composition shifted and bacterial functions like detoxification, dark oxidation, oil bioremediation, cellulolysis, degradation, and chitinolysis were found more frequently in algae cultures with aphelid infection (Fig. 5).

\section{Discussion}

As industrial plant operators rarely publicly publish reasons or frequency of algal culture breakdowns, the financial impact of aphelid contaminations is difficult to assess. This could not be assigned at phylum level. Relative abundance is displayed for four culture treatments (open-AI, open+AI, sterile-AI, sterile + AI) over time. ANPR, Allorhizobium-Neorhizobium-Pararhizobium-Rhizobium

study investigated the temporal bacterial community composition of four microalgal cultures under open and sterile culture conditions in presence and absence of aphelid parasites. Distinct bacterial communities for each algae species were observed, which only sparsely shifted over time in different cultivation conditions. Once aphelids were present, bacterial diversity increased, and algae rapidly lost their chlorophyll and biomass regardless if sterile laboratory conditions or nonsterile culture conditions were applied. Moreover, aphelid infection changed the bacterial functional profiles, which implicate a fundamental breakdown in the bacterial-algae interaction patterns.

\section{Algae species-specific bacterial microbiota}

Bacterial community compositions associated with algal hosts have previously been characterized by the dominance 


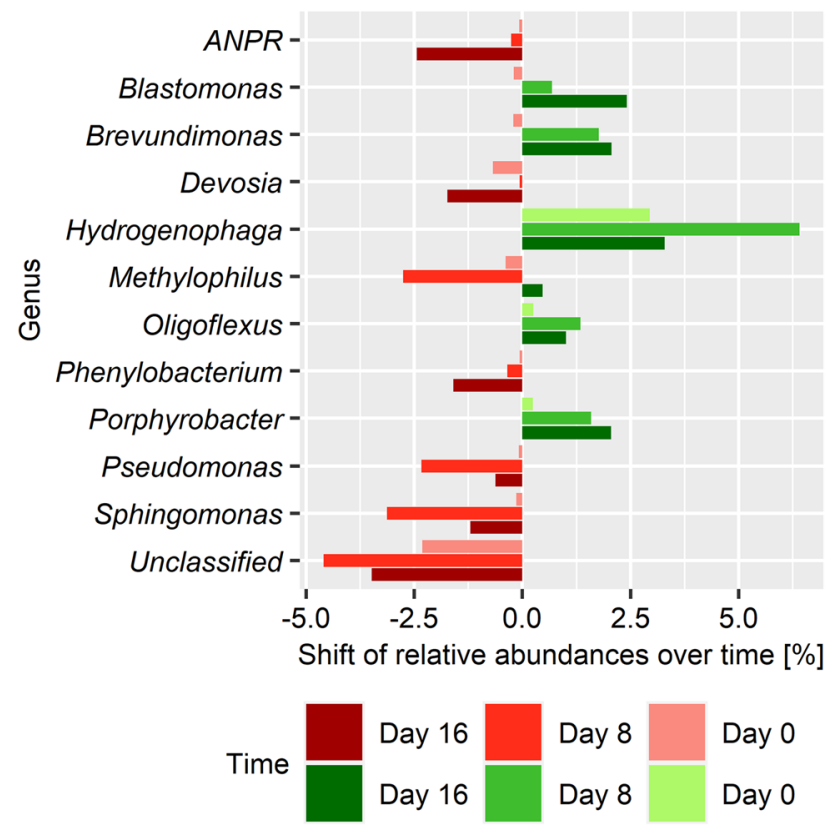

Fig. 4 Shift in the relative abundances of bacterial genera retrieved from infected $S$. vacuolatus cultures (+ AI) incubated under open and sterile culture conditions over time. Negative shift in relative abundances of respective bacterial genus (red) indicate increased abundance in the sterile culture conditions, while positive shift (green) indicate increased abundance in the open culture conditions. ANPR, Allorhizobium-Neorhizobium-Pararhizobium-Rhizobium

of Proteobacteria, Bacteroidetes, and Actinobacteria (Lakaniemi et al. 2012b; Krohn-Molt et al. 2013; Ramanan et al. 2015; Geng et al. 2016b; Fulbright et al. 2018), which is in line with our findings on phylum level. However, previous studies were inconclusive on species specificity of phycosphere bacteria on finer taxonomic levels (Sapp et al. 2007; Ramanan et al. 2015). In this study, bacterial microbiomes were significantly adapted to their algal hosts at genus level (Figs. 2 and 3). Although most bacterial genera occurred frequently in varying abundances in all algae species, we have found distinct and stable abundance patterns for each algal host species. These findings support the idea of a distinct core microbiome with a close relationship to its algal host, which was recently supported by findings for Chlorella saccharophila, Scenedesmus quadricauda, and Micrasterias crux-melitensis and its associated bacterial community members (Krohn-Molt et al. 2017). Such implications were also suggested for epibiotic microbiota-diatom community patterns, which were observed as long-term associations in controlled laboratory conditions (Crenn et al. 2018). Moreover, Ramanan et al. (2015) concluded that overarching bacterial functional traits are essential for the maintenance of biogeochemical networks in the nutrient-rich phycosphere, which also directly affects algae livelihood and bacterial-algal interaction patterns.

\section{Effects of culture conditions on bacterial communities}

Furthermore, it is still under discussion, whether associated microbiomes in artificial algae cultures originate from sampling habitats and might be preserved for years in strain culture collections or whether bacteria might be invading axenic strain cultures while nonsterile upscaling procedures were carried out.

Our findings clearly showed the presence of persistent algae-specific microbiomes from the beginning of the experiments, irrespective of open or sterile culture conditions. Precultures from Koethen's strain collection have been handled with or without contact to the open environment in experimental preparation and upscaling, so immigration of new bacterial strains was very likely only in open culture conditions. However, open conditions had no impact on the algal growth and in most cases only a minor impact on the bacterial community composition (Figs. 1,2, and 3), indicating that invasion of nonalgal specific microbiome members is less successful. Such maintenance in the bacterial core microbiome indicates a high
Fig. 5 Bacterial functional diversity in infected $(+\mathrm{AI})$ and noninfected (-AI) algae cultures. The presence of each predicted function was calculated for every sample belonging to both sample groups and deviations between groups over $10 \%$ were displayed as a bar plot and were based on FAPROTAX according to Louca et al. (2016). More information to the functions can be found in Supplementary Table S4

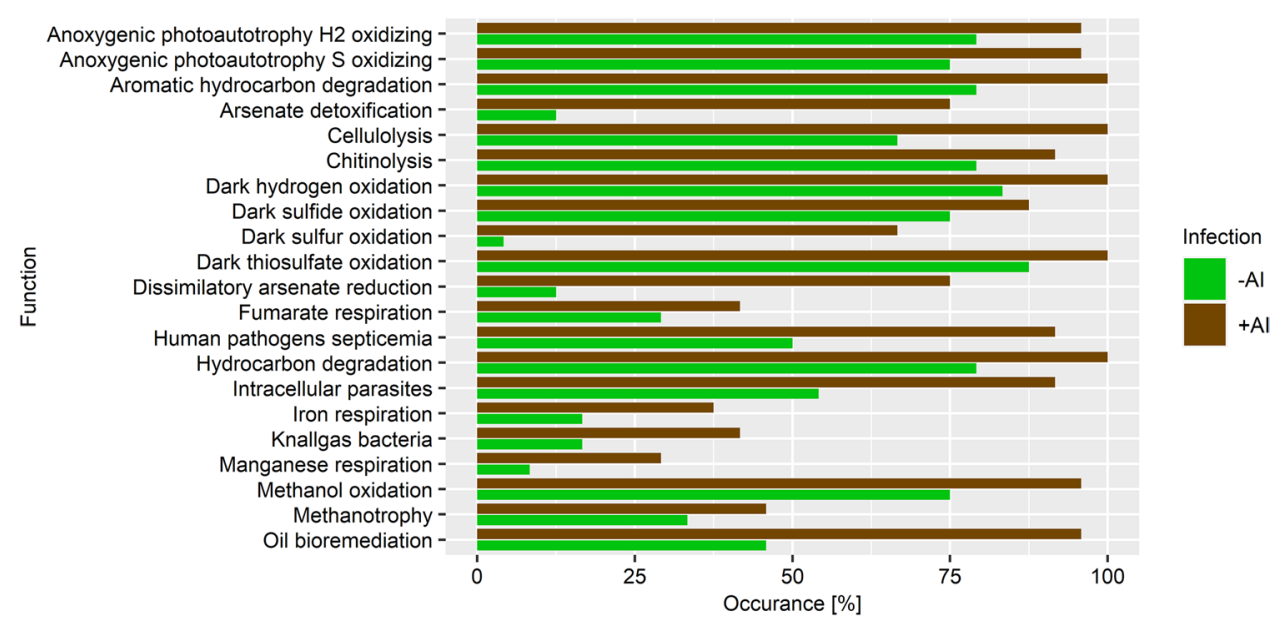


degree of adaption and robustness to the specific holobiont. However, since the bacterial community composition of $S$. vacuolatus exhibited the most tremendous changes over time, more incubation time points were analyzed to understand the succession of the bacterial compositions (Fig. S5). Members of the genus Brevundimonas, which dominated open cultures, were previously found to promote the growth of algal hosts (Park et al. 2008). Members of the genus Devosia and Blastomonas dominating the sterile cultures of $S$. vacuolatus were described with high transcription activity in the biofilm of a Scendesmus species linked to algae effector molecules (Krohn-Molt et al. 2017). These findings indicate that shifts in the algal culture conditions were associated with some bacterial genera that support algal growth in changing culture conditions. After acclimatization of the algal host, a broader spectrum in the matured bacterial community composition was established which supplement the abundant members of the core microbiome. In turn, the presence of aphelid parasites triggered a different and more diverse bacterial pattern of the algal host S. vacuolatus (Fig. 3, Fig. S5). The results of multiple long-term outdoor studies also suggested the persistence of a core microbiome but, in contrast to our experiments, observed seasonal changes (Biondi et al. 2017) or growth phase dependent changes of parts of the community (Geng et al. 2016b; Fulbright et al. 2018). Behringer and colleagues (2018) compared the composition of short-term cultivation of natural diatom isolates and long-term cultivation of strain collection cultures of diatoms. Results of this study suggest that in controlled laboratory culture conditions, interspecies interactions select for a specific, but simplified microbial community, shaped by long-term associations of diatoms and bacteria (Behringer et al. 2018). A recent study focused on the question of how bacterial community assembly processes are organized in the microalgal phycosphere of Phaeodactylum tricornutum and Microchloropsis salina in outdoor raceway ponds and laboratory enrichments (Kimbrel et al. 2019). Results showed that algal-associated bacterial communities were not only shaped by deterministic processes like algal host interactions and culture conditions, as reported in our study, but also by stochastic processes like initial inoculum composition of the algal microbiome.

\section{Impact of parasitic infections on bacterial communities}

The effects of zoosporic parasites in algae cultivation systems for industrial applications are, so far, poorly assessed even though these interactions were known from natural environments (Gerphagnon et al. 2013; Grossart et al. 2016; Frenken et al 2017). This study assessed how indigenous bacterial microbiomes of algae cultures were affected by an aphelid parasitosis. Not only algae growth was inhibited by the infection, but the bacterial community was also clearly afflicted in this process. Bacterial diversity increased (Fig. 3), and the community composition was altered over the course of aphelid infection in structure and functional profiles (Figs. 2, 3, and 5). Bacterial functions related to biomass breakdown were detected more often in infected algal cultures compared to noninfected ones (Fig. 5). This may result from an increasing number of dead algae biomass that was released by aphelids and became bioavailable.

There are some reports that bacterial community composition can be altered by antibiotic treatment and quorum sensing compounds (Geng et al. 2016a) and under the attack of ciliates (Lakaniemi et al. 2012a), but to our knowledge, there is only very few data published that specifically focuses on the effect of algae parasites on associated microbial communities in industrial cultivation systems. For instance, one study by Carney et al. (2014) monitored the dynamics of the microbiome in a microalgal mass culture growing in municipal wastewater. They reported an algal biomass decline by chytrid parasitosis related to a simultaneous shift in the bacterial community composition (from $\gamma$-Proteobacteria to Bacteroidetes) in a municipal wastewater photobioreactor, supporting our hypothesis that parasitic infections not only impact the algal population but also affect the associated microbial community.

\section{Conclusions}

Our study showed robust and temporal stable community patterns of the algae host and its associated microbiome were shifted once algal hosts were invaded by aphelids. Moreover, the functional traits of these microbiomes also shifted from algae-cooperative to decomposing traits. So far, algal target sites for initial aphelid invasion are unknown; therefore, more research is needed to understand how algae target sites can be protected and/or the aphelid actions can be disturbed or weakened by algae-bacterial interactions (McBride et al., 2016). Upcoming scientific research should address the microbial-algal interaction patterns during aphelid infection at the proteomic level in order to elucidate the cross-kingdom organismal link at the physiological level. Moreover, the advantage of temporal and spatial diversification of algal crops should be addressed, which may mitigate algal loss in case of an aphelid infection.

Supplementary Information The online version contains supplementary material available at https://doi.org/10.1007/s10811-021-02542-9 .

Acknowledgements We are grateful to Prof. Dr. Shigeru Okada from the Faculty and Graduate School of Agriculture and Life Science/ 
University of Tokyo for providing the Botryococcus braunii strain var. Showa.

Author contribution ALH designed the study and conducted laboratory work, data analysis, and writing of the manuscript. CG supervised and funded the study and commented on the manuscript. MN contributed to the experimental design, funding, and writing of the manuscript.

Funding Open Access funding enabled and organized by Projekt DEAL. We would like to thank the Ministry of Economy and Science of Saxonia-Anhalt for the financial support of this study.

Data availability Sequencing data is publicly available at NCBI sequence read archive under BioProject accession number PRJNA680549 (https://www.ncbi.nlm.nih.gov/sra/PRJNA680549)

\section{Declarations}

Conflict of interest The authors declare no conflict of interest.

Open Access This article is licensed under a Creative Commons Attribution 4.0 International License, which permits use, sharing, adaptation, distribution and reproduction in any medium or format, as long as you give appropriate credit to the original author(s) and the source, provide a link to the Creative Commons licence, and indicate if changes were made. The images or other third party material in this article are included in the article's Creative Commons licence, unless indicated otherwise in a credit line to the material. If material is not included in the article's Creative Commons licence and your intended use is not permitted by statutory regulation or exceeds the permitted use, you will need to obtain permission directly from the copyright holder. To view a copy of this licence, visit http://creativecommons.org/licenses/by/4.0/.

\section{References}

Andersen RA (ed) (2015) Algal culturing techniques. Elsevier, Amsterdam

Azam F (1998) Microbial control of oceanic carbon flux: the plot thickens. Science 280:694-696

Beckers B, Beeck M, op de, Thijs S, Truyens S, Weyens N, Boerjan W, Vangronsveld J (2016) Performance of 16S rDNA primer pairs in the study of rhizosphere and endosphere bacterial microbiomes in metabarcoding studies. Front Microbiol 7:650

Behringer G, Ochsenkühn MA, Fei C, Fanning J, Koester JA, Amin SA (2018) Bacterial communities of diatoms display strong conservation across strains and time. Front Microbiol 9:659

Biondi N, Cheloni G, Tatti E, Decorosi F, Rodolfi L, Giovannetti L, Viti C, Tredici MR (2017) The bacterial community associated with Tetraselmis suecica outdoor mass cultures. J Appl Phycol 29:67-78

Bischoff HW, Bold HC (1963) Some soil algae from Enchanted Rock and related algal species. Phycological Studies, University of Texas IV:1-95

Buchan A, LeCleir GR, Gulvik CA, González JM (2014) Master recyclers: features and functions of bacteria associated with phytoplankton blooms. Nat Rev Microbiol 12:686-698

Buettner C, Noll M (2018) Differences in microbial key players in anaerobic degradation between biogas and sewage treatment plants. Int Bioder Biodegrad 133:124-132

Carney LT, Lane TW (2014) Parasites in algae mass culture. Front Microbiol 5:278
Carney LT, Reinsch SS, Lane PD, Solberg OD, Jansen LS, Williams KP, Trent JD, Lane TW (2014) Microbiome analysis of a microalgal mass culture growing in municipal wastewater in a prototype OMEGA photobioreactor. Algal Res 4:52-61

Carney LT, Wilkenfeld JS, Lane PD, Solberg OD, Fuqua ZB, Cornelius NG, Gillespie S, Williams KP, Samocha TM, Lane TW (2016) Pond crash forensics: presumptive identification of pond crash agents by next generation sequencing in replicate raceway mass cultures of Nannochloropsis salina. Algal Res 17:341-347

Cirri E, Pohnert G (2019) Algae-bacteria interactions that balance the planktonic microbiome. New Phytol 223:100-106

Cooper MB, Smith AG (2015) Exploring mutualistic interactions between microalgae and bacteria in the omics age. Curr Opin Plant Biol 26:147-153

Crenn K, Duffieux D, Jeanthon C (2018) Bacterial epibiotic communities of ubiquitous and abundant marine diatoms are distinct in short- and long-term associations. Front Microbiol 9:2879

Ding Y, Peng X, Wang Z, Wen X, Geng Y, Li Y (2017) Isolation and characterization of an endoparasite from the culture of oleaginous microalga Graesiella sp. WBG-1. Algal Res 26:371-379

Edgar RC (2004) MUSCLE: multiple sequence alignment with high accuracy and high throughput. Nucleic Acids Res 32:1792-1797

Ferro L, Hu YO, Gentili FG, Andersson AF, Funk C (2020) DNA metabarcoding reveals microbial community dynamics in a microalgae-based municipal wastewater treatment open photobioreactor. Algal Res 51:102043

Frenken T, Alacid E, Berger SA, Bourne EC, Gerphagnon M, Grossart H-P, Gsell AS, Ibelings BW, Kagami M, Küpper FC, Letcher PM, Loyau A, Miki T, Nejstgaard JC, Rasconi S, Reñé A, Rohrlack T, Rojas-Jimenez K, Schmeller DS, Scholz B, Seto K, Sime-Ngando T, Sukenik A, van de Waal DB, van den Wyngaert S, van Donk E, Wolinska J, Wurzbacher C, Agha R (2017) Integrating chytrid fungal parasites into plankton ecology: research gaps and needs. Environ Microbiol 19:3802-3822

Fuentes JL, Garbayo I, Cuaresma M, Montero Z, González-del-Valle M, Vílchez C (2016) Impact of microalgae-bacteria interactions on the production of algal biomass and associated compounds. Mar Drugs 14:100

Fulbright SP, Robbins-Pianka A, Berg-Lyons D, Knight R, Reardon $\mathrm{KF}$, Chisholm ST (2018) Bacterial community changes in an industrial algae production system. Algal Res 31:147-156

Gachon CMM, Sime-Ngando T, Strittmatter M, Chambouvet A, Kim GH (2010) Algal diseases: spotlight on a black box. Trends Plant Sci 15:633-640

Geng H, Tran-Gyamfi MB, Lane TW, Sale KL, Yu ET (2016) Changes in the structure of the microbial community associated with Nannochloropsis salina following treatments with antibiotics and bioactive compounds. Front Microbiol 7:1155

Geng H, Sale KL, Tran-Gyamfi MB, Lane TW, Yu ET (2016) Longitudinal analysis of microbiota in microalga Nannochloropsis salina cultures. Microb Ecol 72:14-24

Gerphagnon M, Latour D, Colombet J, Sime-Ngando T (2013) Fungal parasitism: life cycle, dynamics and impact on cyanobacterial blooms. PLoS ONE 8:e60894

Gibbs RA, Hayes CR (1988) The use of R2A medium and the spread plate method for the enumeration of heterotrophic bacteria in drinking water. Lett Appl Microbiol 6:19-21

Gromov B, Mamkaeva KA (1968) Amoeboaphelidium protococcarum sp. n. and Amoeboaphelidium chlorellavorum sp. n.- endoparasites of protococcous algae. Acta Protozoologica:224-225

Gromov B, Mamkaeva KA (1969) Sensitivity of different Scenedesmus strains to the endoparasitic microorganism Amoeboaphelidium. Phycologia 7:19-23

Grossart H-P, Wurzbacher C, James TY, Kagami M (2016) Discovery of dark matter fungi in aquatic ecosystems demands a reappraisal 
of the phylogeny and ecology of zoosporic fungi. Fungal Ecol 19:28-38

Gurung TB, Urabe J, Nakanishi M (1999) Regulation of the relationship between phytoplankton Scenedesmus acutus and heterotrophic bacteria by the balance of light and nutrients. Aquat Microb Ecol 17:27-35

Hoffman Y, Aflalo C, Zarka A, Gutman J, James TY, Boussiba S (2008) Isolation and characterization of a novel chytrid species (phylum Blastocladiomycota), parasitic on the green alga Haematococcus. Mycol Res 112:70-81

Jephcott TG, van Ogtrop FF, Gleason FH, Macarthur DJ, Scholz B (2017) The ecology of chytrid and aphelid parasites of phytoplankton. In: Dighton J, White JF (eds) The Fungal Community. CRC Press, Boca Raton, pp 239-256

Kagami M, Miki T, Takimoto G (2014) Mycoloop: chytrids in aquatic food webs. Front Microbiol 5:166

Karpov SA, Mamkaeva MA, Aleoshin VV, Nassonova E, Lilje O, Gleason FH (2014) Morphology, phylogeny, and ecology of the aphelids (Aphelidea, Opisthokonta) and proposal for the new superphylum Opisthosporidia. Front Microbiol 5:112

Karpov SA, Vishnyakov, AE, López-García P, Zorina NA, Ciobanu M, Tcvetkova VS, Moreira D (2020) Morphology and molecular phylogeny of Aphelidium insulamus sp. nov. (Aphelida, Opisthosporidia). https://hal.archives-ouvertes.fr/hal-03043373/ document

Kazamia E, Aldridge DC, Smith AG (2012) Synthetic ecology - a way forward for sustainable algal biofuel production? J Biotechnol 162:163-169

Kazamia E, Helliwell KE, Purton S, Smith AG (2016) How mutualisms arise in phytoplankton communities: building eco-evolutionary principles for aquatic microbes. Ecol Lett 19:810-822

Kim B-R, Shin J, Guevarra R, Lee JH, Kim DW, Seol K-H, Lee J-H, Kim HB, Isaacson R (2017) Deciphering diversity indices for a better understanding of microbial communities. J Microbiol Biotechnol 27:2089-2093

Kimbrel JA, Samo TJ, Ward C, Nilson D, Thelen MP, Siccardi A, Zimba P, Lane TW, Mayali X (2019) Host selection and stochastic effects influence bacterial community assembly on the microalgal phycosphere. Algal Res 40:101489

Koedooder C, Stock W, Willems A, Mangelinckx S, de Troch M, Vyverman W, Sabbe K (2019) Diatom-bacteria interactions modulate the composition and productivity of benthic diatom biofilms. Front Microbiol 10:1255

Krohn-Molt I, Wemheuer B, Alawi M, Poehlein A, Güllert S, Schmeisser C, Pommerening-Röser A, Grundhoff A, Daniel R, Hanelt D, Streit WR (2013) Metagenome survey of a multispecies and alga-associated biofilm revealed key elements of bacterialalgal interactions in photobioreactors. Appl Environ Microbiol 79:6196-6206

Krohn-Molt I, Alawi M, Förstner KU, Wiegandt A, Burkhardt L, Indenbirken D, Thieß M, Grundhoff A, Kehr J, Tholey A, Streit WR (2017) Insights into microalga and bacteria interactions of selected phycosphere biofilms using metagenomic, transcriptomic, and proteomic approaches. Front Microbiol 8:1941

Kumar S, Stecher G, Li M, Knyaz C, Tamura K (2018) MEGA X: Molecular evolutionary genetics analysis across computing platforms. Mol Biol Evol 35:1547-1549

Lakaniemi A-M, Hulatt CJ, Wakeman KD, Thomas DN, Puhakka JA (2012a) Eukaryotic and prokaryotic microbial communities during microalgal biomass production. Bioresour Technol 124:387-393

Lakaniemi A-M, Intihar VM, Tuovinen OH, Puhakka JA (2012b) Growth of Chlorella vulgaris and associated bacteria in photobioreactors. Microb Biotechnol 5:69-78

Larsson J (2020) eulerr: area-proportional Euler and venn diagrams with ellipses. $\mathrm{R}$ package version 6.1 .0
Le Boulch M, Déhais P, Combes S, Pascal G (2019) The MACADAM database: a MetAboliC pAthways DAtabase for microbial taxonomic groups for mining potential metabolic capacities of archaeal and bacterial taxonomic groups. Database. https:// doi.org/10.1093/database/baz049

Letcher PM, Powell MJ (2019) A taxonomic summary of Aphelidiaceae. IMA Fungus 10:4

Letcher PM, Lopez S, Schmieder R, Lee PA, Behnke C, Powell MJ, McBride RC (2013) Characterization of Amoeboaphelidium protococcarum, an algal parasite new to the cryptomycota isolated from an outdoor algal pond used for the production of biofuel. PLoS ONE 8:e56232

Letcher PM, Longcore JE, Quandt A, da Silva Leite D, James TY, Powell MJ (2017) Morphological, molecular, and ultrastructural characterization of Rozella rhizoclosmatii, a new species in Cryptomycota. Fungal Biology 121:1-10

Lian J, Wijffels RH, Smidt H, Sipkema D (2018) The effect of the algal microbiome on industrial production of microalgae. Microb Biotechnol 11:806-818

Louca S, Wegener Parfrey L, Doebeli M (2016) Decoupling function and taxonomy in the global ocean microbiome. Science 353:1268-1272

Matthes S, Matschke M, Cotta F, Grossmann J, Griehl C (2015) Reliable production of microalgae biomass using a novel microalgae platform. J Appl Phycol 27:1755-1762

Mayers TJ, Bramucci AR, Yakimovich KM, Case RJ (2016) A bacterial pathogen displaying temperature-enhanced virulence of the microalga Emiliania huxleyi. Front Microbiol 7:892

McBride RC, Lopez S, Meenach C, Burnett M, Lee PA, Nohilly F, Behnke C (2014) Contamination management in low cost open algae ponds for biofuels production. Indust Biotechnol 10:221-227

McBride RC, Smith VH, Carney LT, Lane TW (2016) Crop protection in open ponds. In: Slocombe SP, Benemann JR (eds) Microalgal production for biomass and high-value products. CRC Press, Boca Raton

McMurdie PJ, Holmes S (2013) phyloseq: an R package for reproducible interactive analysis and graphics of microbiome census data. PLoS ONE 8:e61217

Mendes LBB, Vermelho AB (2013) Allelopathy as a potential strategy to improve microalgae cultivation. Biotechnol Biofuels 6:152

Moheimani NR, Borowitzka MA, Isdepsky A, Fon Sing S (2013) Standard methods for measuring growth of algae and their composition. In: Borowitzka MA, Moheimani NR (eds) Algae for Biofuels and Energy. Springer, Dordrecht, pp 265-284

Mönnich J, Tebben J, Bergemann J, Case R, Wohlrab S, Harder T (2020) Niche-based assembly of bacterial consortia on the diatom Thalassiosira rotula is stable and reproducible. ISME J 14:1614-1625

Oksanen J, Blanchet FG, Friendly M, Kindt R, Legendre P, Mcglinn D, Minchin PR, O'Hara RB, Simpson GL, Solymos P, Stevens MHH, Szoecs E, Wagner H (2019) Vegan: community ecology package. R package version 2.4-2. Community Ecology Package 2.5-6:1-296

Park Y, Je K-W, Lee K, Jung S-E, Choi T-J (2008) Growth promotion of Chlorella ellipsoidea by co-inoculation with Brevundimonas sp. isolated from the microalga. Hydrobiologia 598:219-228

Piwosz K, Vrdoljak A, Frenken T, González-Olalla JM, Šantić D, McKay RM, Spilling K, Guttman L, Znachor P, Mujakić I, Fecskeová LK, Zoccarato L, Hanusová M, Pessina A, Reich T, Grossart H-P, Koblížek M (2020) Light and primary production shape bacterial activity and community composition of aerobic anoxygenic phototrophic bacteria in a microcosm experiment. mSphere 5. https://doi.org/10.1128/mSphere.00354-20 
Pruesse E, Peplies J, Glöckner FO (2012) SINA: accurate highthroughput multiple sequence alignment of ribosomal RNA genes. Bioinformatics 28:1823-1829

Ramanan R, Kang Z, Kim BH, Cho DH, Jin L, Oh HM, Kim HS (2015) Phycosphere bacterial diversity in green algae reveals an apparent similarity across habitats. Algal Res 8:140-144

Ramanan R, Kim B-H, Cho D-H, Oh H-M, Kim H-S (2016) Algaebacteria interactions: evolution, ecology and emerging applications. Biotechnol Adv 34:14-29

Rasconi S, Grami B, Niquil N, Jobard M, Sime-Ngando T (2014) Parasitic chytrids sustain zooplankton growth during inedible algal bloom. Front Microbiol 5:229

Saitou N, Nei M (1987) The neighbor-joining method: a new method for reconstructing phylogenetic trees. Mol Biol Evol 4:406-425

Sansupa C, Wahdan SFM, Hossen S, Disayathanoowat T, Wubet T, Purahong W (2021) Can we use functional annotation of prokaryotic taxa (FAPROTAX) to assign the ecological functions of soil bacteria? Appl Sci 11:688

Sapp M, Schwaderer AS, Wiltshire KH, Hoppe H-G, Gerdts G, Wichels A (2007) Species-specific bacterial communities in the phycosphere of microalgae? Microb Ecol 53:683-699

Schenk PM, Thomas-Hall SR, Stephens E, Marx UC, Mussgnug JH, Posten C, Kruse O, Hankamer B (2008) Second generation biofuels: high-efficiency microalgae for biodiesel production. BioEnergy Res 1:20-43

Schnepf E, Hegewald E, Soeder C-J (1971) Elektronenmikroskopische Beobachtungen an Parasiten aus Scenedesmus-Massenkulturen. Arch Microbiol 75:209-229

Scott SA, Davey MP, Dennis JS, Horst I, Howe CJ, Lea-Smith DJ, Smith AG (2010) Biodiesel from algae: challenges and prospects. Currt Opin Biotechnol 21:277-286

Seto K, Matsuzawa T, Kuno H, Kagami M (2020) Morphology ultrastructure and molecular phylogeny of Aphelidium collabens sp nov Aphelida a parasitoid of a green alga Coccomyxa sp. Protist 171:125728

Seyedsayamdost MR, Wang R, Kolter R, Clardy J (2014) Hybrid biosynthesis of roseobacticides from algal and bacterial precursor molecules. J Am Chem Soc 136:15150-15153
Seymour JR, Amin SA, Raina J-B, Stocker R (2017) Zooming in on the phycosphere: the ecological interface for phytoplankton-bacteria relationships. Nat Microbiol 2:17065

Shurin JB, Abbott RL, Deal MS, Kwan GT, Litchman E, McBride RC, Mandal S, Smith VH (2013) Industrial-strength ecology: trade-offs and opportunities in algal biofuel production. Ecol Lett 16:1393-1404

Sime-Ngando T (2012) Phytoplankton chytridiomycosis: fungal parasites of phytoplankton and their imprints on the food web dynamics. Front Microbiol 3:361

Smith VH, Crews T (2014) Applying ecological principles of crop cultivation in large-scale algal biomass production. Algal Res 4:23-34

Tedersoo L, Sánchez-Ramírez S, Kõljalg U, Bahram M, Döring M, Schigel D, May T, Ryberg M, Abarenkov K (2018) High-level classification of the Fungi and a tool for evolutionary ecological analyses. Fungal Diversity 90:135-159

Torruella G, Grau-Bové X, Moreira D, Karpov SA, Burns JA, SebéPedrós A, Völcker E, López-García P (2018) Global transcriptome analysis of the aphelid Paraphelidium tribonemae supports the phagotrophic origin of fungi. Commun Biol 1:231

van Donk E (1989) The role of fungal parasites in phytoplankton succession. In: Sommer U (ed) Plankton Ecology: Succession in Plankton Communities. Springer, Berlin, pp 171-194

Waddell PJ, Steel MA (1997) General time-reversible distances with unequal rates across sites: mixing gamma and inverse Gaussian distributions with invariant sites. Mol Phylogenet Evol 8:398-414

Wang H, Zhang W, Chen L, Wang J, Liu T (2013) The contamination and control of biological pollutants in mass cultivation of microalgae. Bioresour Technol 128:745-750

Wang H, Hill RT, Zheng T, Hu X, Wang B (2014) Effects of bacterial communities on biofuel-producing microalgae: stimulation, inhibition and harvesting. Crit Rev Biotechnol 36:341-352

Publisher's note Springer Nature remains neutral with regard to jurisdictional claims in published maps and institutional affiliations. 